\title{
Kernos
}

Revue internationale et pluridisciplinaire de religion grecque antique

7| 1994

Varia

\section{Le type iconographique de la statue cultuelle d'Apollon Amyklaios : un emprunt oriental ?}

\section{Eleni Georgoulaki}

\section{(apenEdition \\ Journals}

\section{Édition électronique}

URL : http://journals.openedition.org/kernos/1100

DOI : 10.4000/kernos. 1100

ISSN : 2034-7871

\section{Éditeur}

Centre international d'étude de la religion grecque antique

\section{Édition imprimée}

Date de publication : 1 janvier 1994

ISSN : 0776-3824

\section{Référence électronique}

Eleni Georgoulaki, «Le type iconographique de la statue cultuelle d'Apollon Amyklaios : un emprunt oriental ? », Kernos [En ligne], 7 | 1994, mis en ligne le 20 avril 2011, consulté le 19 avril 2019. URL http://journals.openedition.org/kernos/1100; DOI : 10.4000/kernos.1100 
Kernos, 7 (1994), p. 95-118.

\section{LE TYPE ICONOGRAPHIQUE DE LA STATUE CULTUELLE D'APOLLON AMYKLAIOS : UN EMPRUNT ORIENTAL ?*}

\section{Données archéologiques, sources philologiques}

Le sanctuaire d'Apollon Amyklaios se trouvait sur la colline d'Aghia Kyriaki (Fig. 1), sur le site d'Amyclées, à une distance de $5 \mathrm{~km}$ au sud de la ville moderne de Sparte. Un culte y était établi dès l'époque mycénienne (milieu du XIIIe siècle av. J.-C.), comme le montrent des trouvailles assez importantes (Pl. I), semblables à celles faites dans d'autres sanctuaires contemporains ${ }^{1}$; cependant, on constate qu'à la fin de l'Âge du Bronze, les offrandes ${ }^{2}$ ont été interrompues pendant une période de 100 à 150 ans : selon Aikaterini Dimakopoulou ${ }^{3}$, le sanctuaire mycénien étant presque abandonné à cause de la pauvreté de l'époque, de nouveaux habitants sont arrivés sur le site, porteurs de la céramique protogéométrique (Pl. II : deuxième moitié du Xe siècle av. J.-C.), et y ont établi un nouveau culte, celui d'Apollon.

D'après les fouilles, l'existence d'un culte est attestée pendant plusieurs siècles ${ }^{4}$, de l'époque protogéométrique jusqu'à l'époque romaine. En plus, au début de l'époque archaïque, on fait construire la

* Je voudrais remercier le conseil d'administration du CIERGA, qui m'a permis de participer à ce colloque; je voudrais aussi remercier Mlle Fabienne Pironet qui a corrigé mon français, le photographe de l'Université de Liège, M. Botty, pour les photos qu'il a prises, et M. le Professeur R. Laffineur pour sa bienveillante attention.

1 Le matériel de l'époque mycénienne - tout en argile - est constitué de 71 statuettes féminines de type $\Psi$, de 2 statuettes de cavaliers, de 28 vases de taureaux, de 32 statuettes d'animaux, d'un oiseau, de la partie supérieure de la tête d'une femme avec polos, d'une main humaine avec une kylix et de 6 fragments de vases; le matériel du sanctuaire, à son entier, était publié par

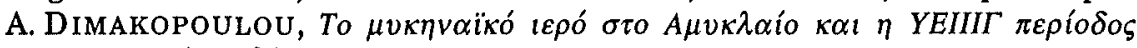
$\sigma \tau \eta v \Lambda \alpha \kappa \omega v i ́ \alpha, A \theta \dot{\eta} v \alpha, 1982$.

2 EAD., op. cit., p. 35

3 EAD., op. cit., p. 9, 35

4 E. Buschor - W. VON MASSOW, Vom Amyklaion, in MDAI(A), 52 (1927), p. 1-85, pl. VI 
statue cultuelle d'Apollon et un peu plus tard, le trône du dieu (Fig. 2). D'après le témoignage de Pausanias ${ }^{5}$ - le seul auteur qui ait décrit la statue et le trône -, la statue est vieille et grossière, et, à l'exception du visage, des mains et de la pointe des pieds, elle ressemble à une colonne; la tête est casquée et le dieu porte un arc et une lance dans les mains. On peut supposer qu'elle fut réalisée selon la technique de sphyrelaton, c'està-dire qu'un corps de bois était couvert de bandes de bronze ${ }^{6}$. Cette image du dieu se retrouve sur des monnaies spartiates de l'époque romaine ${ }^{7}$, mais sa date exacte reste inconnue : la technique de sphyrelaton fut introduite au VIIIe siècle av. J.-C. mais, étant donné que les premiers exemples de sculpture monumentale datent de la fin du VIIe siècle av. J.-C., on pourrait proposer une date plus tardive pour notre statue, probablement le cours du VIIe siècle av. J.-C. ${ }^{8}$ Avant la fin du VIe siècle av. J.-C., le sculpteur Bathyclès a construit le trône du dieu, dont la base était considérée comme la tombe d'Hyakinthos; l'apothéose de ce dernier apparaissait en relief sur des plaques de marbre (Fig. 2). Des fragments du trône ont été découverts pendant la fouille du site en 1892 par C. Tsountas et en 1904 par A. Furtwängler 9 .

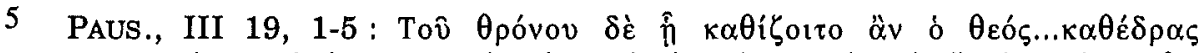

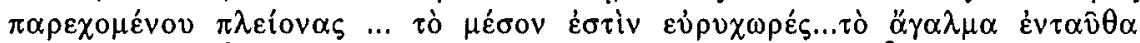

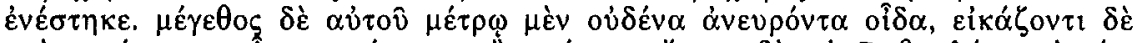

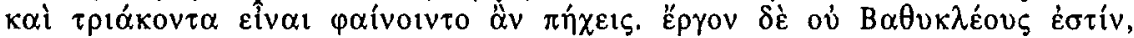

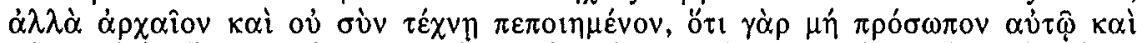

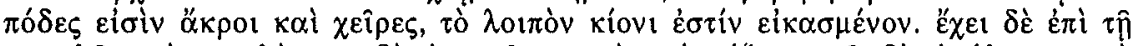

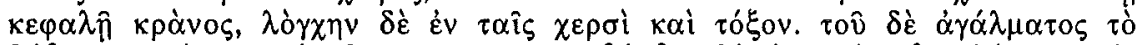

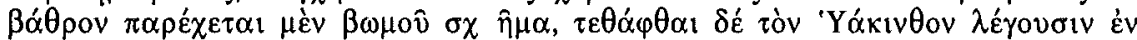

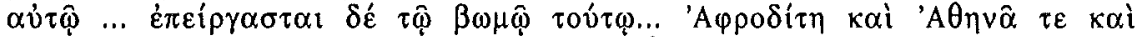

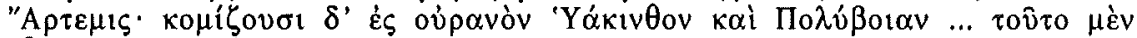

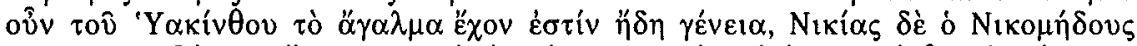

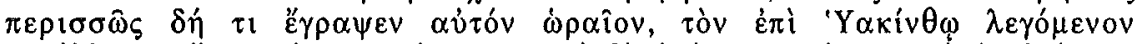

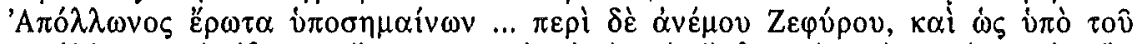

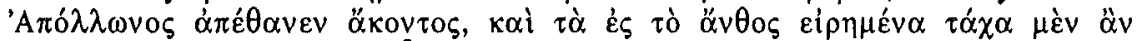

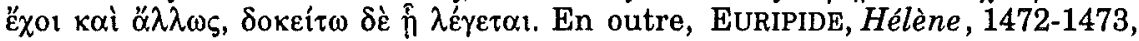
est le temoignage le plus ancien sur l'amour d'Apollon pour Hyakinthos; ATHÉNÉE, IV, 139c-f, décrit la fête d'Hyakinthia.

6 Cf. M. Pettersson, Cults of Apollo at Sparta, Göteborg, 1992 (ActAth. in- $8^{\circ}$, 12), p. 11, n. 16.

7 S. Grunauer von Hoerschelmann, Die Münzrägung der Lakedaimonier, Berlin, 1978, p. 99, pl. 27 : XLVII : 4, XLVIII: 2, 28: LVI: 5, 32:12; cf. J. ROMANO, Early Greek cult images, Diss. Univ. of Pennsylvania, 1980, p. 100.

8 J. ROMANO, op. cit., p. 104

9 C. Tsountas, Ex tov A $\mu v \kappa \lambda \alpha$ íov, in $A E$ (1892), p. 1-26; E. FieCHTER, Amyklae.

Der Thron des Apollon, in JDAI, 33 (1918), p. 107-245. 


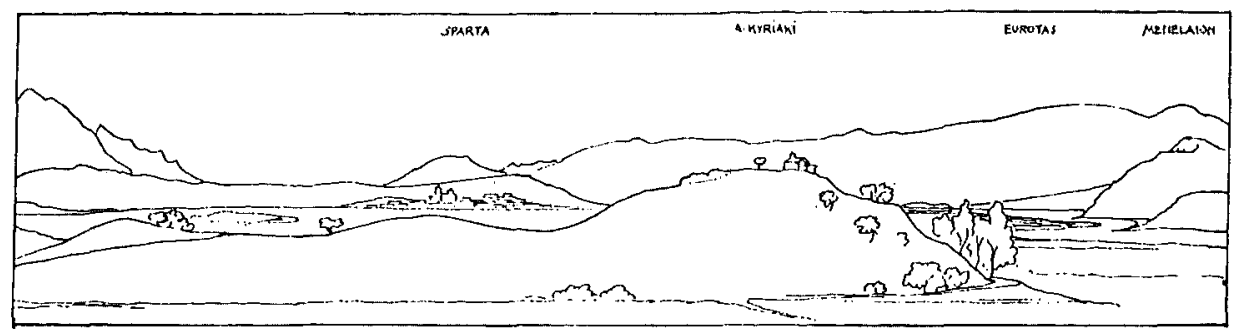

Fig. 1 : Le site d'Amyclées

d'après E. FiECHTER, JDAI, 33 (1918), fig. 1
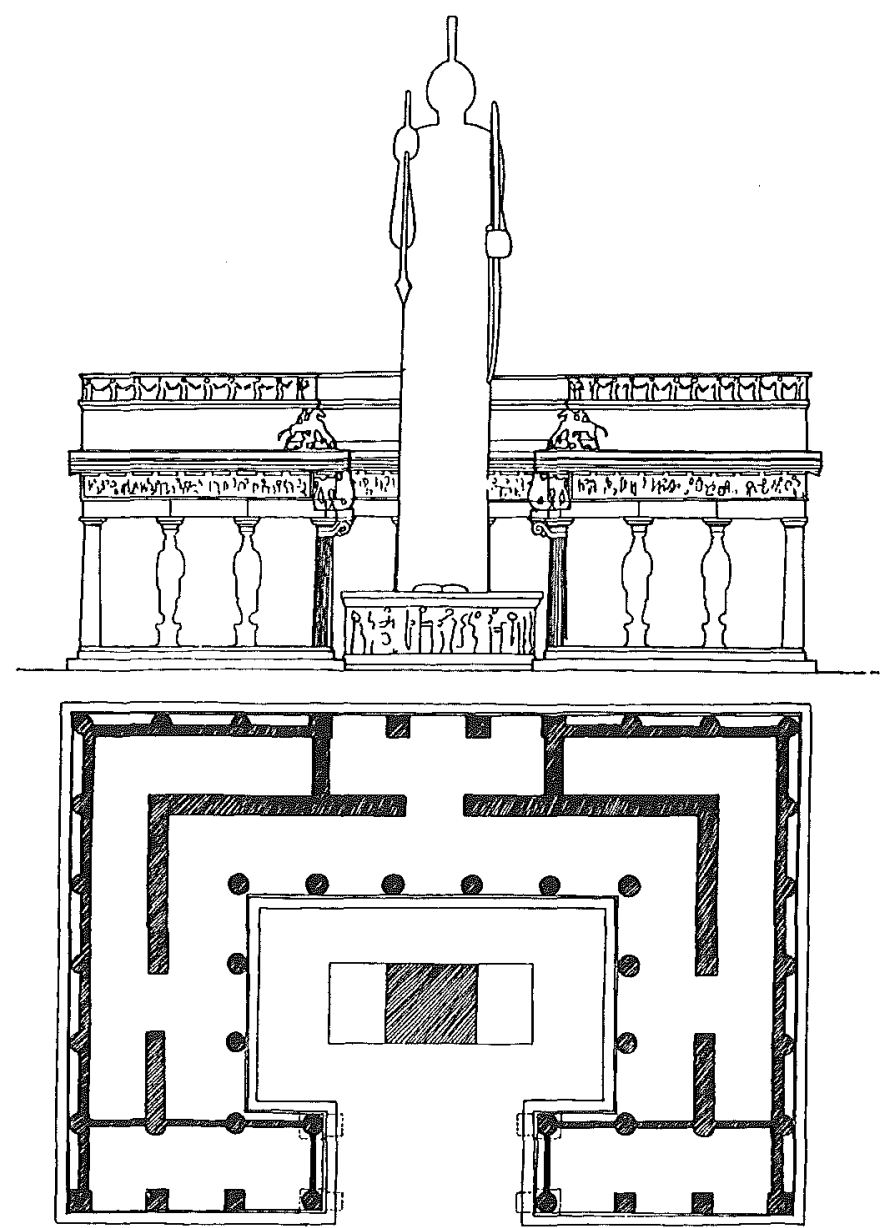

Fig. 2 : Apollon Amyklaios, reconstruction d'après, E. BuSCHOR - W. VON MASSOW, MDAI (A) 52 (1927), p. 18 


\section{Interprétation - Suggestions}

Aikaterini Dimakopoulou ${ }^{10}$, qui a examiné le matériel, constate que le sanctuaire d'Amyclées a été fondé à l'époque mycénienne et réutilisé à partir de l'époque protogéométrique; en réalité, on ne peut mettre en rapport la période MRIIIC avec le Xe siècle av. J.-C. parce qu'il n'y a aucune relation stylistique entre les deux rythmes de la céramique provenant de l'Amyklaion. M. Pettersson ${ }^{11}$, par contre, insiste sur le fait qu'il n'y aurait pas d'interruption du culte entre les deux périodes et que la tradition de productivité des vases en argile serait restée vivante depuis l'époque mycénienne jusqu'à l'Âge du Fer; cependant, $P$. Cartledge ${ }^{12}$ et $W$. Coulson ${ }^{13}$ argumentent en faveur de la distinction de ces deux styles.

Hyakinthos : Bien que le nom d'Hyakinthos soit absent des tablettes en Linéaire $B$, le témoignage de Pausanias ${ }^{14}$ nous laisse supposer qu'il s'agit d'un ancien dieu local dont la mémoire a survécu à l'époque postmycénienne; ensuite, à l'époque archaïque, on a fait construire un autel-tombe en son honneur. N. Papachatzis ${ }^{15}$ croit que Hyakinthos aurait été une divinité chthonienne pendant l'Âge du Bronze et que, lors de l'introduction d'Apollon comme divinité principale du site, il serait devenu un simple héros divinisé dont l'apothéose est représentée sur le trône d'Apollon ${ }^{16}$.

Apollon : Le nom d'Apollon n'apparaît pas non plus sur les tablettes en Linéaire B; le type "pajawone» (au Paijon) sur une tablette de Knossos (KN V52 ${ }^{17}$ ) est une appellation récente d'Apollon: dans l'Iliade

10 A. Dimakopoulou, op. cit., p. 32.

11 M. Pettersson, op. cit., p. 97, 99.

12 P. CARTLEdge, Sparta and Lakonia. A regional history 1300-362 BC, London, 1979 , p. 92.

13 W.D.E. CouLson, The Dark age pottery of Sparta, in ABSA, 80 (1985), p. 64, 65.

14 PAUS., III, 19, 4.

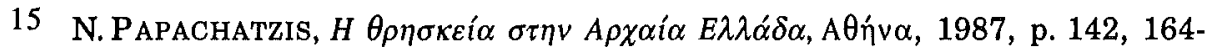
166, 194.

16 Discussion et références bibliographiques sur le culte d'Hyakinthos,

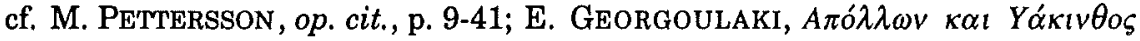

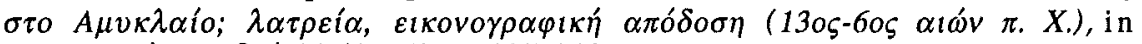

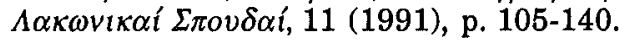

17 M. GÉRARD-ROUSSEAU, Les mentions religieuses dans les tablettes mycéniennes, Roma, 1968 (Inculabula Graeca 29), p. 164-165. 


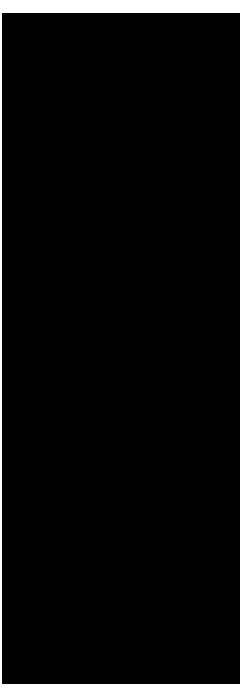

1

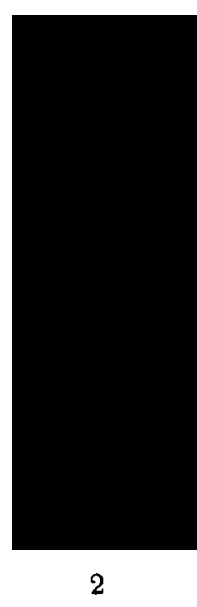

Fig. 3: Trouvailles protogéométriques en métal

d'après E. BUSCHOR - W. VON MASSOW, MDAI (A) 52 (1927), fig. 17

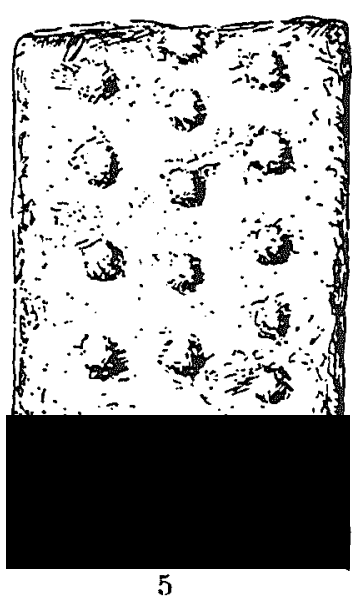

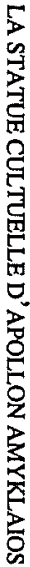


(A, 473; D, 401), et même chez Hésiode (fr. 307), il n'y a aucun rapport entre les deux divinités; le syncrétisme entre eux a probablement eu lieu après l'époque homérique, en même temps que la systématisation du panthéon ${ }^{18}$.

D'après les trouvailles (Fig. 3 : poterie, offrandes en métal) ${ }^{19}$, la date exacte de l'introduction du culte d'Apollon à Amyclées n'est pas certaine; certes, les vases protogéométriques pourraient indiquer l'arrivée d'un nouveau peuple, mais ce peuple serait-il aussi porteur d'idées religieuses nouvelles? $M$. Pettersson ${ }^{20}$ croit qu'Apollon serait plutôt un dieu des communautés qui se sont développées dans la région à la suite de la chute du monde mycénien. Néanmoins, un nouvel élément dans le culte d'Amyclées est le rapprochement de deux divinités masculines : il n'y a pas de témoignage d'amours divines homosexuelles à l'époque créto-mycénienne; au contraire, les scènes pédérastiques sont plus nombreuses dans l'iconographie archaïque ${ }^{21}$.

Les premières images d'Apollon datent du VIIIe siècle av. J.-C. ${ }^{22}$, mais on n'en a pas découvert à Amyclées. En réalité, C. Tsountas ${ }^{23}$ avait trouvé la tête d'une statue d'argile casquée (hauteur préservée : $8 \mathrm{~cm}$; hauteur initiale $: \pm 40 \mathrm{~cm}$ ) de la deuxième moitié du VIIIe siècle av. J.-C. (Pl. III); vu la hauteur totale de l'image, on pourrait penser à une image d'Apollon ${ }^{24}$. Pourtant, l'absence des attributs du dieu et l'existence d'une tête féminine contemporaine de la tête masculine et provenant du même sanctuaire ${ }^{25}$ rendent la théorie plutôt improbable : si l'une des deux représente Apollon, à quelle déesse l'autre pourrait-elle

18 M. PetTersson (op. cit., p. 20, n. 92) croit que le syncrétisme a eu lieu au début du VIIIe siècle av. J.-C.

19 Op. cit. (n. 2).

20 M. PetTersson, op. cit., p. 97

21 S. KAEMPF-DimitrIADOU, Die Liebe der Götter in der attischen Kunst des 5. Jahrhunderts $v$. Chr., in $A K, 11$ (1979), p. 14-16, 80-81, pl. 6-7; discussion cf. M. PETTERSSON, op. cit., p. 30-35.

22 Lexicon Iconographicum Mythologiae Classicae, II 1, Zürich, 1984, p. 183 sq.

23 C. Tsountas, op. cit., pl. $4: 4$.

24 Suggestion des K. PFEIFF, Apollon: die Wandlung seines Bildes in der Griechischen Kunst, Frankfurt, 1943, p. 21-22; H. WALTER, Griechische Götter: Ihr Gestaltwandel aus den Bewustseinsstufen des Menschen dargestellt an den Bildwerken, München, 1971, p. 327.

25 E. KUNZE, $Z u$ den Anfängen der griechischen Plastik, in MDAI(A), 55 (1930), Beilage XLII-III 
être attribuée ? À Artémis ? Pourtant, à l'Amyklaion on vénérait deux dieux masculins, aucune déesse. En plus, dans le sanctuaire d'Athéna Alea à Tégée ${ }^{26}$, une statuette de guerrier en bronze, découverte avec d'autres offrandes, ressemble, quant aux yeux, à la tête masculine de l'Amyklaion; il s'agit probablement de produits d'artistes locaux, qui connaissaient à tout le moins quelques conventions de l'iconographie du guerrier; ainsi, les deux têtes de l'Amyklaion pourraient être des offrandes de la part d'un guerrier et d'une femme.

Concernant la statue archaïque d'Apollon décrite par Pausanias, il y deux questions à poser :

1) Pourquoi un Apollon armé serait-il mis en rapport avec une divinité chthonienne comme Hyakinthos ? Plutarque ${ }^{27}$ dit que, comme preuve de leurs qualités guerrières, les Spartiates représentaient toutes leurs divinités, masculines et féminines, armées; de plus, le type iconographique d'Apollon Amyklaios, armé d'un arc et d'une lance, très rarement imité, ne le fut jamais en dehors de la Laconie ${ }^{28}$. Il est dès lors probable que ce type soit une création de l'esprit spartiate.

2) Pourquoi Apollon est-il représenté armé d'un arc et d'une lance ? À première vue, cette combinaison semble incompréhensible, mais selon V. Lambrinoudakis ${ }^{29}$, qui a étudié la totalité de l'iconographie du dieu, elle indique un stade intermédiaire entre l'image du guerrier avec la lance de l'époque géométrique et celle de l'archer nu. N'oublions pas qu'Apollon ${ }^{30}$, un peu plus tard, à la fin du VIIe siècle, est représenté armé d'un arc et de flèches, mais la position statique et le casque sont

26 M. VoyatzIs, The Early Sanctuary of Athena Alea at Tegea and other Archaic Sanctuaries in Arcadia, Göterborg, 1990 (SIMA Pocket book 97), p. 103, 116117, 126.

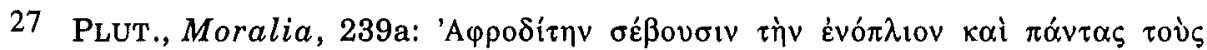

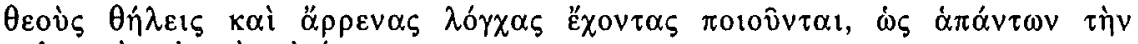

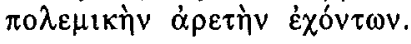

28 a) Monnaies spartiates de l'époque romaine (cf. op. cit.n. 8).

b) Relief en pierre d'Amyclées du IIle siècle av. J.-C (références bibliographiques : LIMC, op. cit. , p. 314).

c) Statue cultuelle d'Apollon Pythaeus à Thornax, un site au N.E. de Sparte,

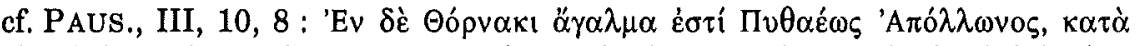

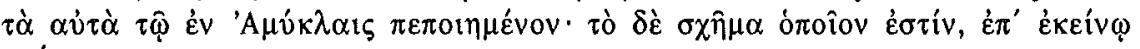
$\gamma \rho \alpha ́ \alpha \omega$.

LIMC, ibidem.

30 LIMC, op. cit. , n ${ }^{\circ} 38,41,43$. 
des caractéristiques des statuettes en bronze du tout début de l'époque géométrique ${ }^{31}$.

\section{Théorie de Burkert}

Pourtant, récemment, W. Burkert a élaboré une théorie sur l'origine orientale de cette statue. Selon lui ${ }^{32}$, les Grecs du VIIIe siècle av. J.-C. ont imité des statuettes d'un dieu guerrier oriental de l'Âge du Bronze, pour représenter certaines de leurs divinités principales : il s'agit d'un homme (Fig. 4) qui porte une ceinture et un chapeau pointu assez haut, il est armé (lance, bouclier) et souvent cornu - signe de sacralité.

Ce type iconographique correspond à des divinités variées de Syrie, d'Égypte et de Palestine, mais W. Burkert penche plutôt pour le dieu Resep des inscriptions égyptiennes. En effet, des dédicaces à cette divinité, datées des VIIe-IIIe siècles av. J.-C., ont été dégagées à Chypre, et des statuettes de ce type ont été importées en Grèce dès l'époque mycénienne.

L'hypothèse de $W$. Burkert repose sur trois faits : 1) il existe une statuette du VIIe siècle av. J.-C. de l'Acropole d'Athènes ${ }^{33}$ qui ressemble aux exemples orientaux quant au chapeau cônique et à la lance (perdue),

31 Archives de l'Institut Archéologique Allemand : «Bronze-Inst. negativ 73/ 1014, 73/ 1015, 73/ 1016 [Kreta, Herakleion Museum Inv. ? (hauteur : $10 \mathrm{~cm}$ )] : Geometrische Bronzestatuette mit Helm von H. Triada" (non publiée). M. BYRNE [The Greek Geometric Warrior Figurine, Louvain-la-Neuve 1991 (Archaeologia Transantlantica, 10), p. 14-15, 36-37, 157, 199] a récemment étudié les figurines de guerriers de l'époque géometrique et constate que l'influence orientale sur l'art géometrique est indiscutable; pourtant, les figurines représentant des guerrieurs, qui sont des importations orientales, mais qui ont été trouvées en territoire hellénique, appartiennent à l'époque mycénienne et les figurines orientales provenant du Moyen Orient appartiennent au début du IIe millénaire. Par conséquent, les figurines de l'époque géometrique ne peuvent pas être considérées comme dérivant directement de ces objets importés. M. Byrne démontre, avec des exemples iconographiques à l'appui, que le motif du guerrier à l'époque géometrique a subi l'influence de la Crète minoenne parce que là-bas «the armed figurine with raised hand, a gesture which already had a long tradition in the Minoan salute, continued into Cretan protogeometric and geometric, and was to find its reflection into mainland geometric art».

32 W. BURKert, Resep-Figuren, Apollon von Amyklai und die Erfindung des Opfers auf Cypern. Zur Religiongeschichte der Dunklen Jahrhunderten, in $G B, 4$ (1975), p. 52-75.

33 M. VOYATZIS, op. cit., fig. 304. 


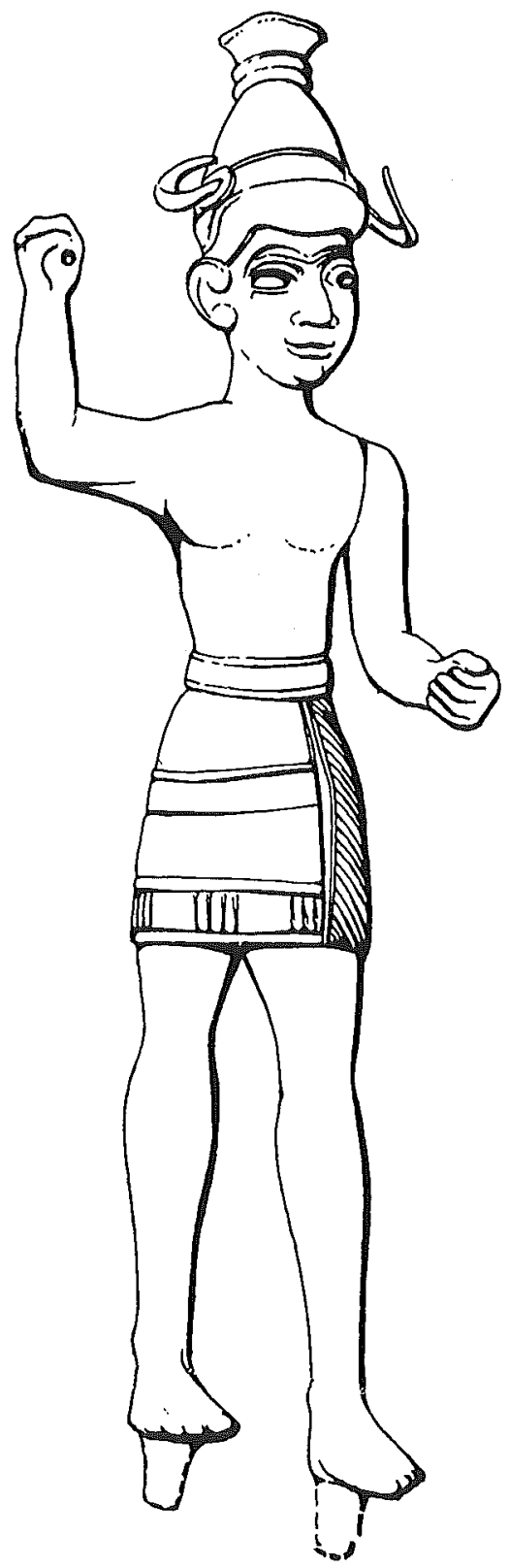

Fig. 4 : Statuettes du type Reshef, provenant d'Orient d'après O. NEGBI, Canaanite Gods in Metal, 1976, fig. 42-50 
mais diffère d'eux, parce qu'elle est nue; 2) elle est de la même hauteur $( \pm 40 \mathrm{~cm})$ que la statuette d'argile de l'Amyklaion; 3$)$ on a retrouvé à l'Amyklaion un fragment du corps d'un guerrier de l'époque archaïque $^{34}$. Il en conclut d'une part qu'une statuette de type oriental d'Apollon existait à Amyclées au VIIIe siècle av. J.-C. et que, d'autre part, l'importation de statuettes orientales en Grèce (résultat de pillages) au XIIe siècle av. J.-C. a eu pour conséquence que les Grecs du VIIIe siècle av. J.-C. ont utilisé le type iconographique du guerrier ${ }^{35}$ comme prototype pour leurs statues cultuelles monumentales, même si, au niveau idéologique, il y avait des différences entre les dieux grecs et ceux de l'Orient.

En plus, à Enkomi de Chypre, deux statues masculines cornues du XIIe siècle av. J.-C. ont été identifiées comme étant des images d'Apollon - plus tard un Apollon Kepalớm a été vénéré à Chypre et, selon une inscription bilingue d'Idalion des IVe-IIIe siècles av. J.-C., Apollon "A $\mu \cup \kappa \lambda \circ \varsigma_{\zeta}$ était assimilé à Reshef-Mkl-; ainsi, selon W. Burkert, on pourrait assimiler l'Apollon Amyklaios et l'Apollon

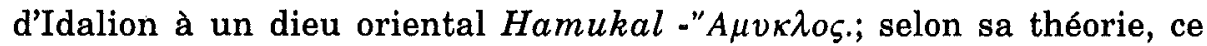
dieu existait déjà à l'époque mycénienne mais les Doriens ont changé son nom de Reshef en 'A $\pi \hat{e} \lambda \lambda \omega v /$ 'A $\pi$ ó $\lambda \lambda \omega v$.

L'hypothèse finale de $W$. Burkert est qu'au XIIe siècle av. J.-C., période de décadence du monde mycénien, à Chypre, un Paijon crétomycénien a été assimilé à un dieu Syrien Resep (A)mukal; ce culte, comme les statuettes du dieu de la guerre, a été introduit dans le Péloponnèse, où le dieu a été transformé en une divinité dorienne.

\section{Données archéologiques - Réfutation de la théorie de W. Burkert}

1) Concernant l'élément créto-mycénien d'Apollon, on a déjà démontré que ce n'est qu'à l'époque archaïque que Paijon et Apollon étaient assimilés.

2) W. Burkert appelle Apollon la tête en argile et le fragment du corps en provenance de l'Amyklaion, mais il ne donne aucune justification à cela. Il compare aussi la tête en argile avec la statuette de l'Acropole

34 E. BUSCHOR - W. VON MASSOW, op. cit., fig. 21.

35 Ora NEGBI aussi (Evidence for Early Phoenician Communities on th Eastern Mediterranean Islands, in Levant, 1982, p. 179-182) croit, à cause de la découverte de deux statuettes du type Reshef dans le sanctuaire de Phylakopi à Milos et à Patsos de Crète, qu'un dieu sémitique aurait été introduit en Égée. 
d'Athènes, à cause de la similitude des linéaments du visage et du "chapeau". Ces traits sont cependant plus caractéristiques de l'époque que d'une divinité.

3) Les statuettes du type Reshef ${ }^{36}$ en Orient représentaient des divinités variées ${ }^{37}:$ l'Addad akkadien (dieu de l'orage) est assimilé au dieu aramaïque $\mathrm{Hadad}$, assimilé à son tour au dieu de Canaan $\mathrm{Baal}^{38}$ qui est une divinité céleste et souveraine. En outre, un autre dieu de Canaan, Rasap (sur des inscriptions d'Ugarit, il s'appelle $r-s-p$ ), est considéré comme dieu guerrier et chthonien en Syrie, mais en Égypte, il a aussi un aspect de fécondité. Ce Rasap égyptien est assimilé ${ }^{39}$ au dieu de Mésopotamie Nergal - dieu de la puissance destructrice, de la nature et de la fécondité en même temps.

Ce serait vraiment difficile d'identifier Apollon à une de ces divinités, à partir de qualités communes (par exemple, dieu du soleil et sauveur, dieu guerrier et chthonien), car toutes ces qualités appartiennent à plusieurs divinités préhistoriques.

Cependant, W. Burkert suggère que l'influence orientale sur les statues grecques est plutôt sensible au niveau iconographique. En effet, sur un sceau syrien 40 de l'Âge du Bronze moyen (Pl. IV), Nergal (identifié par une inscription cunéiforme) porte une lance et un arc (les armes d'Apollon Amyklaios); étant donné que Nergal était assimilé au Reshef et qu'un Reshef $m-k-l$ était assimilé à Apollon Amyklos sur une inscription chypriote de l'époque classique, on pourrait prêter, selon W. Burkert, l'origine du type iconographique de la statue cultuelle

36 Analyse : H. SEeden, The Standing Armed Figurines in the Levant, München, 1980 (Prähistorische Bronzefunde, I, 1).

37 P. Poupard (éd.), Dictionnaire des Religions, Paris, 1984, p. 135, 1194, 1231; cf. R. StadelmanN, Syrisch-Palästinische Gottheiten in Ägypten, Leiden, 1967, p. 47-55.

38 H. SEEDEN, op. cit. , p. 149.

39 Dans un catalogue des divinités babyloniennes, provenant de la bibliothèque du dernier roi d'Ugarit Hammourabi; cf. J. NOUGAYROL, E. Laroche, C. Virolleaud, C. SCHAEFFer, Ugaritica $V$, nouveaux textes accadiens, hourrites et ugaritiques des archives et bibliothèques privées d'Ugarit commentaires des textes historiques (Ire partie), Paris, 1967, p. 42 sq.

40 L. Delaporte, Catalogue des cylindres orientaux du Musée du Louvre, Paris, II, 1923, A919 pl. 96 : 17 (cf. l'inscription : "Irianni, fils de Wate...ni, serviteur de Nergal»); cf., l'opinion de H. SEEDEN, op. cit., p. 148-149 : «No metal figurine from the Levant shows the warrior god armed with the bow". 
d'Apollon Amyklaios au Nergal du sceau syrien; mais, dans ce cas, les armes de Nergal, plus tard assimilé à Reshef, auraient "voyagé» à partir des XVIIe-XVIe siecles av. J.-C. et se seraient «installées» au VIIe siècle av. J.-C. à Amyclées; pourtant, les stades intermédiaires de ce long voyage, qui a duré presque 1000 ans, restent inconnus. En plus, Janice Crowley ${ }^{41}$, qui a étudié les influences orientales sur l'art égéen, constate que les Mycéniens n'ont pas adopté le motif du «dieu menaçant» parce que le symbolisme oriental de celui-ci n'avait aucun sens pour eux. Mais s'il n'y a pas eu d'influence à l'Âge du Bronze, il est improbable de supposer que ce phénomène a pu avoir lieu quatre siècles plus tard.

En réalité, 13 statuettes ${ }^{42}$ en bronze du type Reshef (Fig. 5) ont été trouvées sur le territoire hellénique : 11 d'entre elles datent de l'époque mycénienne et furent découvertes dans des sanctuaires (Délos, Phylacopi de Milos), des habitats (Rhodes, Mycènes, Thermon) ou dans un contexte inconnu (Crète, Attique, Thessalie, Tiryns); 2 seulement ont été trouvées dans des contextes du VIIe siècle av. J.-C., une au sanctuaire de Poséidon à Sounion et l'autre à l'Héraion de Samos.

Ce matériel dispersé au niveau chronologique et géographique autorise plusieurs interprétations : ces statuettes ont été considérées comme héritages ${ }^{43}$, comme propriétés ${ }^{44}$ d'étrangers installés en Grèce, comme amulettes ${ }^{45}$ ou encore comme reproductions du dieu masculin mycénien ${ }^{46}$. Étant donné le nombre limité du matériel, en disproportion avec le grand nombre d'interprétations proposées, on pourrait

41 J. Crowley, The Aegean and the East, Jonsered, 1989 (SIMA Pocket book 51), p. $245,278$.

42 Références bibliographiques, cf. H. SEEDEN, op.cit., p. 125 sq.; C. LAMBRoUPhillipson, Hellenorientalia, The Near Eastern presence in the Bronze Age Aegean, ca. 3000-1100 BC, Göterborg, 1990 (SIMA Pocket book 95), p. 74, 226, 321, 361; H. GALLET DE SANTERRE, Les statuettes de bronze mycéniennes au type dit du 'Dieu Reshef' dans leur contexte Égéen, in $B C H, 111$ (1987), p. 10 13.

43 J. CROWLEY, op. cit., p. 247.

44 C. Lambrou Phillipson, op. cit., p. 155.

45 H. SEEDEN, op. cit., p. 131.

46 St. HILler, Mycenean Traditions in Early Greek Cult Images, in The Greek Rennaissance of the 8th century B.C. Proceedings of the Second International Symposium at the Swedish Institute at Athens (1-5 June 1981), Stockholm, 1983, p. 93. 
accepter l'opinion de H. Gallet de Santerre ${ }^{47}$ qu'il ne s'agirait pas d'importations orientales et qu'il est difficile de trouver une réponse définitive au problème de leur utilisation sur le territoire hellénique.

Quant au sanctuaire d'Amyclées, les découvertes n'attestent aucun contact avec l'Orient à l'époque mycénienne; de plus, les deux exemples de statuettes du type Reshef qui datent de l'époque historique proviennent de Samos et d'Attique; ainsi, il semble impossible de justifier une influence de l'Orient à Amyclées, tant au niveau artistique que cultuel.

\section{Données de Chypre}

W. Burkert considère qu'Apollon Reshef a été transplanté à Amyclées via l'île de Chypre. En effet, des trouvailles 48 archéologiques prouvent un contact entre Chypre et l'Orient à l'époque mycénienne et 44 objets chypriotes ont été dégagés en Attique, Béotie, Eubée, Argolide, Messénie et dans les îles de l'Égée; par contre, on ne constate aucun contact, même commercial, entre Chypre et le site d'Amyclées.

Quant aux statuettes du type Reshef, on en a trouvé 14 sur le territoire chypriote $^{49}$, dont 8 datent de l'époque mycénienne et 6 de l'époque archaïque; en tout cas, étant donné les relations entre Chypre et l'Orient ${ }^{50}$, on pourrait supposer que des produits locaux empruntaient des caractéristiques iconographiques orientales comme, par exemple, les deux statuettes cornues ${ }^{51}$ du XIIe siècle av. J.-C. dégagées dans deux sanctuaires différents d'Enkomi et mentionnées aussi par W. Burkert.

L'une d'elles ${ }^{52}$ est une statue très naturaliste (Fig. 6), en bronze (hauteur : 52, $4 \mathrm{~cm}$ ), représentant un homme qui porte un casque cornu et

47 H. GALLET DE SANTERRE, art. cit., p. 16, 20.

48 C. Lambrou Phillipson, op. cit., p. 46, 92, chap. 10.

49 H. SEEDEN, op. cit., p. 122-125.

50 ID., op. cit. , p. 122, 131

51 A.B. KNAPP, Copper Production and Divine Protection, Archaeology, Ideology and Social Complexity in Bronze Age Cyprus, Göterborg, 1986 (SIMA Pocket book 42), p. 9-11, 13-14.

52 P. DIKAIOS, The Bronze Statuette of a horned god from Enkomi, in AA (1962), p. 1-39; P. DikaIos, Enkomi; Excavations 1948-1958, (IIIa), Mainz am Rhein, 1971, p. 295, Pl. 139-144; H.W. Catling, Cypriot Bronzework in the Mycenaean World, Oxford, 1964, p. 255-256, n 6, pl. 1; K. HADJIOANNOU, On the identification of the Horned God of Enkomi-Alasia, in C.F.A. SCHAEFFER, Alasia I, Mission Archéologique d'Alasia 4, Paris, 1971, p. 33-42; 

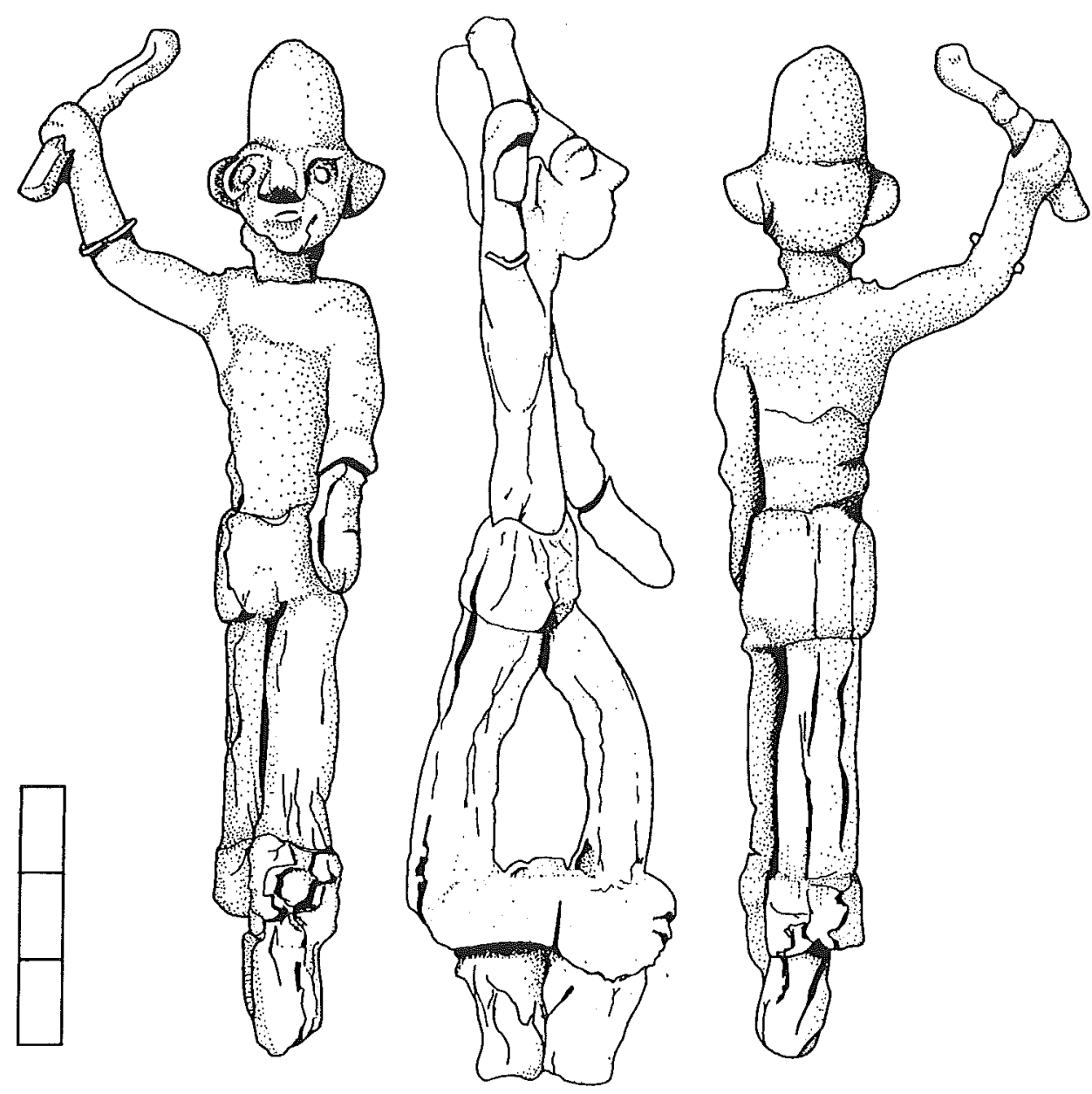

Fig. 5 : Statuettes du type Reshef du sanctuaire mycénien à Phylakopi d'après C. RENFREW, The Sanctuary at Phylakopi, fig. 8

H.G. BuChHolz - V. KARAGEORGHIS, Prehistoric Greece and Cyprus, London, 1973, p. 163, 478, no 1740; M. B URDAJEWITZ, The Sanctuary of the horned god reconsidered, in Archaeologia Cypria I (1985), p. 9-14. 
une jupe courte, l'une de ses mains étant serrée et posée sur sa poitrine. Il y a sur l'identité de cette statue ainsi que sur ses influences possibles plusieurs propositions, dont la plupart sont contradictoires : on l'a identifiée à Nergal ${ }^{53}$, au protecteur d'Alasia ${ }^{54}$, à Apollon 55-Reshef

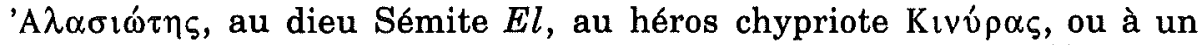
guerrier-adorant. On a parlé aussi des influences égéennes ${ }^{56}$, orientales ${ }^{57}$, des prototypes minoens ${ }^{58}$, des linéaments grecs ou des traditions chypriotes ${ }^{59}$. Parmi cette richesse d'opinions, W. Burkert a repris une théorie développée par P. Dikaios comme base pour sa propre théorie : en effet, P. Dikaios ${ }^{60}$ avait d'abord identifié la statue à Nergal pour ensuite lui préférer Apollon $\mathrm{K} \varepsilon \rho \alpha \alpha_{\alpha} \tau \eta \zeta$, dieu de la fécondité mentionné sur une inscription de $\Lambda \dot{\alpha} \rho v \alpha \kappa \alpha(\Pi v \dot{\lambda} \alpha)$ du IIIe siècle

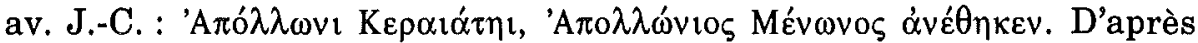
Dikaios, le culte de ce dieu aurait été transplanté à Chypre par des colons achéens d'Arcadie, où il était considéré comme un dieu agraire local. D'après Pausanias 61, il y avait en Arcadie un sanctuaire d" $A \pi o ́ \lambda \lambda \omega v$

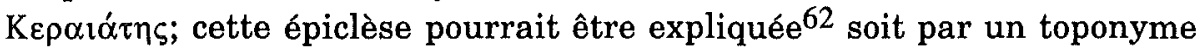

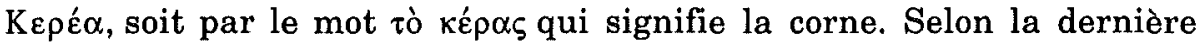
hypothèse, Pausanias aurait fait une faute d'orthographe ${ }^{63}$ et Apollon

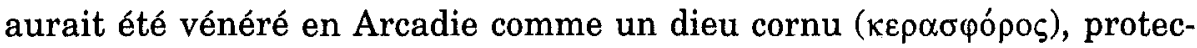

53 P. DIKaIos, The oldest known representation of Apollo, in ILN, 27-8-1949, p. 316-317.

54 C. F. A. SCHAEFFER, The discovery of the ancient capital of Cyprus, in ILN, 208-1949, p. 278-280.

55 H. W. CATling, op. cit. (n. 52).

56 S. MARINATOS, $O K \varepsilon \rho \alpha t \alpha \dot{\alpha} \eta \varsigma \tau \eta \varsigma^{~}{ }^{\prime} E \gamma \kappa \omega \mu \eta \zeta$, in $A D, 18$ (1963), p. 95-98

57 H. W. CATLING, op. cit. (n. 52).

58 V. KARAGEORGHIS, Kú $\pi \rho \circ$, , A $\theta \dot{v} v \alpha, 1974$, p. 30.

59 P. DIKAIOS, op. cit. (n. 52).

60 Cf. A.B. KNAPP, op. cit., p. 14.

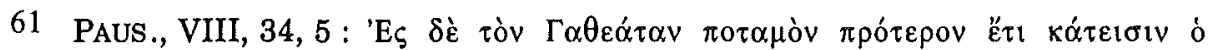

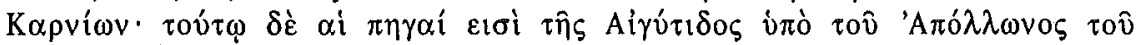

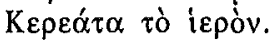

62 M. JoST, Sanctuaires et cultes d'Arcadie, Paris, 1985, p. 482.

63 O. MASSON, Remarques sur les cultes chypriotes à l'époque du Bronze Récent, in The Mycenaeans in the Eastern Mediterranean. Acts of the International Archaeological Symposium, Nicosia, 1973, p. 114, n. 35. 
teur du bétail. Mais, comme Madeleine Jost ${ }^{64}$ l'a démontré, Apollon n'était pas un dieu important en Arcadie; ainsi, il serait vraiment très difficile de supposer qu'un dieu local, plutôt insignifiant, ait voyagé en dehors de son pays d'origine jusqu'à l'île de Chypre et que la mémoire de son culte soit restée vivante pendant 1000 ans jusqu'au IIIe siècle av. J.-C.; pourtant, K. Hadjioannou ${ }^{65}$ accepte la théorie de Dikaios parce qu'Homère décrit Apollon comme un homme costaud et viril, et aussi parce que ses vêtements ressemblent à ceux des statuettes votives de bergers (Pl. V) arcadiens (VIIe-Ve siècle av. J.-C.); mais la ressemblance des vêtements ne justifie pas l'identification avec un dieu local.

La deuxième statuette (Fig. $7:$ hauteur $35 \mathrm{~cm})^{66}$, trouvée dans un autre sanctuaire d'Enkomi, représente un homme barbu armé (lance et bouclier), peut-être masqué, qui porte un casque cornu et une "jupe" jusqu'aux genoux; il se trouve sur une base en forme de lingot - on l'appelle souvent dans la bibliographie «dieu au lingot». Les interprétations ${ }^{67}$ proposées sont une fois encore contradictoires : on l'a identifié à Nergal, à un "guerrier menaçant», à Héphaistos ou à un dieu oriental; on remarque aussi des ressemblances avec des guerriers de Sardaigne ou la présence de caractéristiques syro-palestiniennes.

Après cette analyse, on peut tirer la conclusion que les deux statuettes chypriotes ne représentent pas Apollon et ne sont pas des reproductions exactes des statuettes du type Reshef. En plus, étant donné que des statuettes authentiques de ce type ont été découvertes sur l'île, on peut simplement y constater un syncrétisme d'éléments orientaux et grécomycéniens vers la fin de l'époque mycénienne.

64

65

66 21 (1966), p. 59-69; ID., Alasia I, Mission Archéologique d'Alasia 4, Paris, 1971, p. 506-510; J-C. CouRToIs, Le sanctuaire du dieu au lingot d'Enkomi-Alasia, in Alasia I, op. cit.., pl. XII-XXIVA; ID., Le sanctuaire du dieu au lingot d'EnkomiAlasia (Chypre) et les lieux de culte contemporains en Méditerranée orientale, in CRAI (1973), p. 231-235, pl. 8-9; H.G. BUCHHOLZ - V. KARAGEORGHIS, op. cit., p. 163, 478, $n^{\circ} 1741$; O. NEGBI, Canaanite Gods in Metal: An Archaeological Study of Ancient Syro-Palestinian Figurines, Tel Aviv, 1976, p. 38-39, 168, fig. 151, n 1405; A.B. KNAPP, op. cit., p. 9-11.

67 Références bibliographiques : A.B. KNAPP, op. cit., p. 10. 
Ce syncrétisme est aussi présent sur des inscriptions chypriotes à partir du VIIe siècle av. J.-C. : on y trouve en effet des dédicaces phéniciennes à Reshef, dont 7 à Reshef $M-k-l^{68}$. Une de ces dernières, datée du IVe siècle av. J.-C. et découverte au sanctuaire d'Apollon à Idalion ${ }^{69}$ est bilingue - en écriture phénicienne et chypriote syllabique; il s'agit d'une dédicace au dieu Phénicien Reshef -Mikal, (en fait r-s-p $m-k-l$, ) identifié, dans le texte grec, avec 'A propose-t-il que le dieu d'Amyclées soit assimilé au dieu phénicien Reshef; pourtant, le type $A \mu v \kappa \lambda o s$ est inconnu de la langue grecque, il s'agit plutôt d'une transcription du terme phénicien $m k l$. En plus, sur une autre dédicace à Apollon en langue grecque, provenant du même sanctuaire, on lit l'épiclèse normale 'A $\mu \nu \kappa \lambda \alpha \hat{i}_{0} \varsigma^{70}$. Ainsi peut-on tirer la conclusion que le type $A \mu \nu \kappa \lambda \circ \varsigma$ est une transcription de la langue phéniciennne, mais que le type $A \mu \nu \kappa \lambda \alpha$ lo renvoie à l'Apollon de Laconie .

L'épiclèse $m k l^{71}$ nous autorise à supposer un syncrétisme entre Apollon et Reshef sur le territoire chypriote, mais pas dans le reste de la Grèce, comme le prétend W. Burkert. En outre, il est plus vraisemblable que cette assimilation ${ }^{72}$ ait eu lieu à une époque tardive ${ }^{73}$ et ait été le fait de Grecs qui, arrivant à Chypre, ont constaté quelques aspects communs entre les deux divinités.

\section{Conclusion}

La théorie de W. Burkert ne nous semble pas être justifiée : en effet, il utilise comme base une série d'autres théories, qui elles non plus ne sont pas justifiées, à savoir :

68 A. CaquOT - O. Masson, Deux inscriptions phéniciennes de Chypre, in Syria, 45 (1968), p. 295, 301, 303.

69 O. MASSON, Les inscriptions chypriotes syllabiques, Paris, 1961, p. 246-248

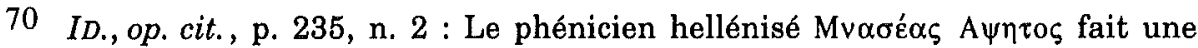
dédicace à Apollon Amyklaios ('A ó$_{\lambda \lambda} \lambda \omega v \mathrm{l}$ 'A $\mu \nu \kappa \lambda \alpha i \omega_{1}$ ).

71 Reshef à Chypre avait aussi, d'autres épiclèses, sauf celle de $m k l$; cf. A. CAQUOT - O. MASSON, op. cit.(n. 39).

72 Cf. l'étude d'E. LIPINSKI, Reshef Amyklos, in Phoenicia and the East Mediterranean in the first millenium B.C., Leuven, 1987 (Studia Phoenicia, 5), p. 87-99.

73 M. Pettersson (op. cit., p. 14, n. 54), même s'il accepte cette assimilation tardive, insiste sur le fait que «the iconographical model of a warrior-god was borrowed by the Greeks from, for example, Cyprus». 
1) Identification d'Apollon et du Paijon créto-mycénien.

2) Identification d'Apollon et de la tête d'argile de l'Amyklaion

3) Identification d'Apollon avec la statuette au lingot chypriote

4) Identification de la statue cornue d'Enkomi avec Apollon Keraiatis

5) Identification d'Apollon Amyklaios et de Reshef Mikal

Il n'y a en outre aucune preuve d'un contact entre Amyclées et l'Orient, ni à l'époque mycéniennne, ni à l'époque historique. Au contraire, à cause de son voisinage avec la Grèce continentale et avec l'Orient, l'île de Chypre était un pays où des assimilations variées (iconographiques et idéologiques) ont eu lieu pendant toute son histoire; ceci pourrait aussi expliquer les influences diverses sur le style de deux statues d'Enkomi et le syncrétisme constaté sur des inscriptions tardives.

Concernant l'Amyklaion, on peut, d'après les trouvailles archéologiques, démontrer l'existence d'un culte à l'époque mycénienne et celle de la statue avec le trône d'Apollon à l'époque archaïque. Cependant, d'après les sources philologiques plus tardives et la tradition du culte sur le lieu, on ne peut formuler que des hypothèses sur l'existence du culte d'Hyakinthos à l'époque mycénienne et sur l'iconographie d'Apollon pendant l'époque géométrique.

Eleni Georgoulaki

Université de Liège

Service d'archéologie grecque

Place du 20-Août, 32

B - 4000 LIÈGE 


\section{Amyklaion}

\begin{tabular}{|l|l|}
\hline \multicolumn{1}{|c|}{$\begin{array}{c}\text { Époque mycéniennne } \\
\text { Données archéologiques }\end{array}$} & \multicolumn{1}{c|}{$\begin{array}{c}\text { Époque historique } \\
\text { Données archéologiques } \\
\text { Sources littéraires }\end{array}$} \\
\hline Statuettes féminines & $\begin{array}{l}\text { Âge du Fer : vases en argile, } \\
\text { dédicaces en métal }\end{array}$ \\
\hline $\begin{array}{l}\text { Statuettes d'animaux } \\
\text { (quadrupèdes, 1 oiseau) }\end{array}$ & $\begin{array}{l}\text { VIIle s. av. J.-C. : Fragments de } \\
\text { deux statues (masculine, fémi- } \\
\text { nine) }\end{array}$ \\
\hline 2 fragments de statues (?) & $\begin{array}{l}\text { VIIe s. av. J.-C. : statue d'Apollon } \\
\text { (semi-iconique, armée) }\end{array}$ \\
\hline Vases de taureaux & $\begin{array}{l}\text { VIes. av. J.-C. : trône d'Apollon } \\
\text { (apothéose d'Hyakinthos) }\end{array}$ \\
\hline 2 statuettes de cavaliers & $\begin{array}{l}\text { Périodes class. et hellénistique : } \\
\text { sources philologiques }\end{array}$ \\
\hline
\end{tabular}

Hypothèses

\begin{tabular}{|l|l|}
\hline \multicolumn{1}{|c|}{ Époque mycénienne } & \multicolumn{1}{c|}{$\begin{array}{c}\text { Époque postmycénienne } \\
\text { Âge du Fer }\end{array}$} \\
\hline $\begin{array}{l}\text { Culte d'Hyakinthos } \\
\text { (sources philologiques) }\end{array}$ & $\begin{array}{l}\text { Introduction du culte d'Apollon } \\
\text { Statue d'Apollon : } \\
\text { type iconographique local }\end{array}$ \\
\hline
\end{tabular}

Théorie de Burkert

\begin{tabular}{|l|l|}
\hline \multicolumn{1}{|c|}{ Identification avec Apollon } & \multicolumn{1}{c|}{ Réfutation } \\
\hline Paijon Créto-mycénien & Données archéologiques \\
\hline $\begin{array}{l}\text { Fragment d'une statue masculine } \\
\text { d'Amyclées }\end{array}$ & Syncrétisme entre Chypre-Orient \\
\hline Statues cornues chypriotes & Pas de contact Amyclées-Chypre, \\
\hline Reshef mkl Hypothèse & Pas de contact Amyclées-Orient \\
\hline \multicolumn{1}{|c|}{ Hyental } & \\
\hline $\begin{array}{l}\text { Type iconographique oriental } \\
\text { pour les statues grecques du VIIIe } \\
\text { siècle av. J.-C. }\end{array}$ & \\
\hline
\end{tabular}



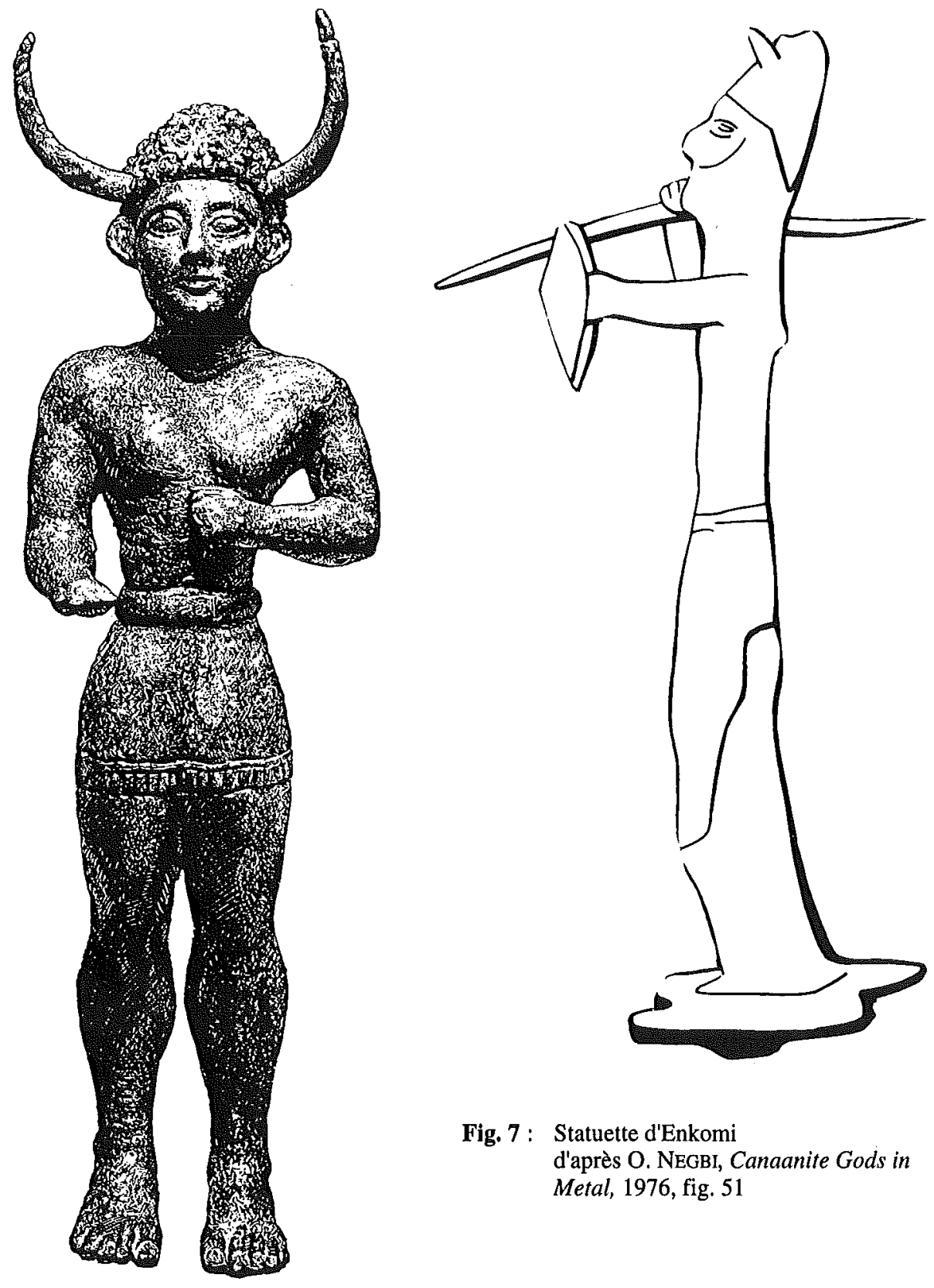

Fig. 7 : Statuette d'Enkomi d'après O. NEGBI, Canaanite Gods in Metal, 1976, fig. 51

Fig. 6: Statuette d'Enkomi d'après P. DIKAIOS, Enkomi, excavations 1948-1958, 1971, pl. 140 

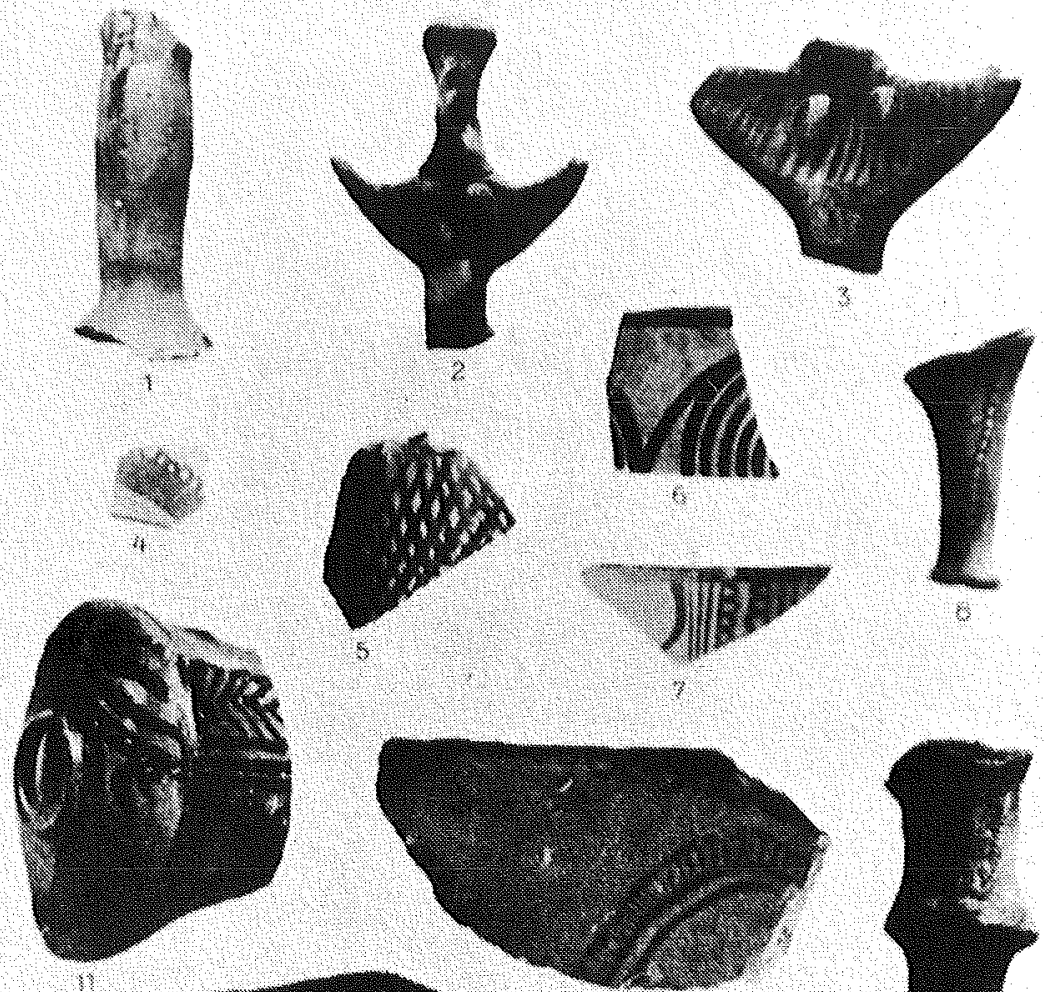

\%

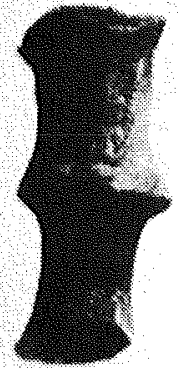

ra

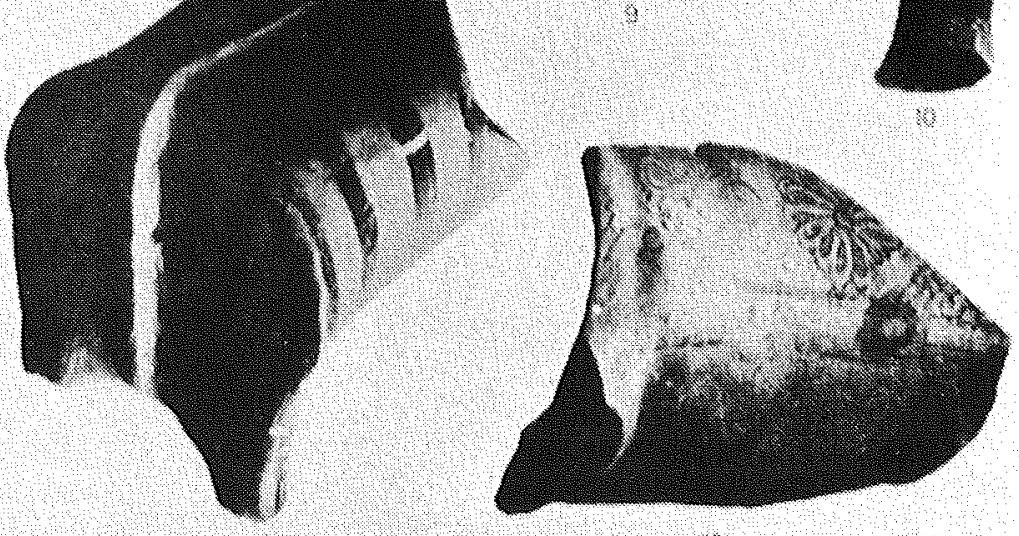

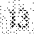

Pl. I : Trouvailles mycéniennes

d'après E. BuSCHOR-W. vON MASSOW, in MDAI(A) 52 (1927), Beilage VI 


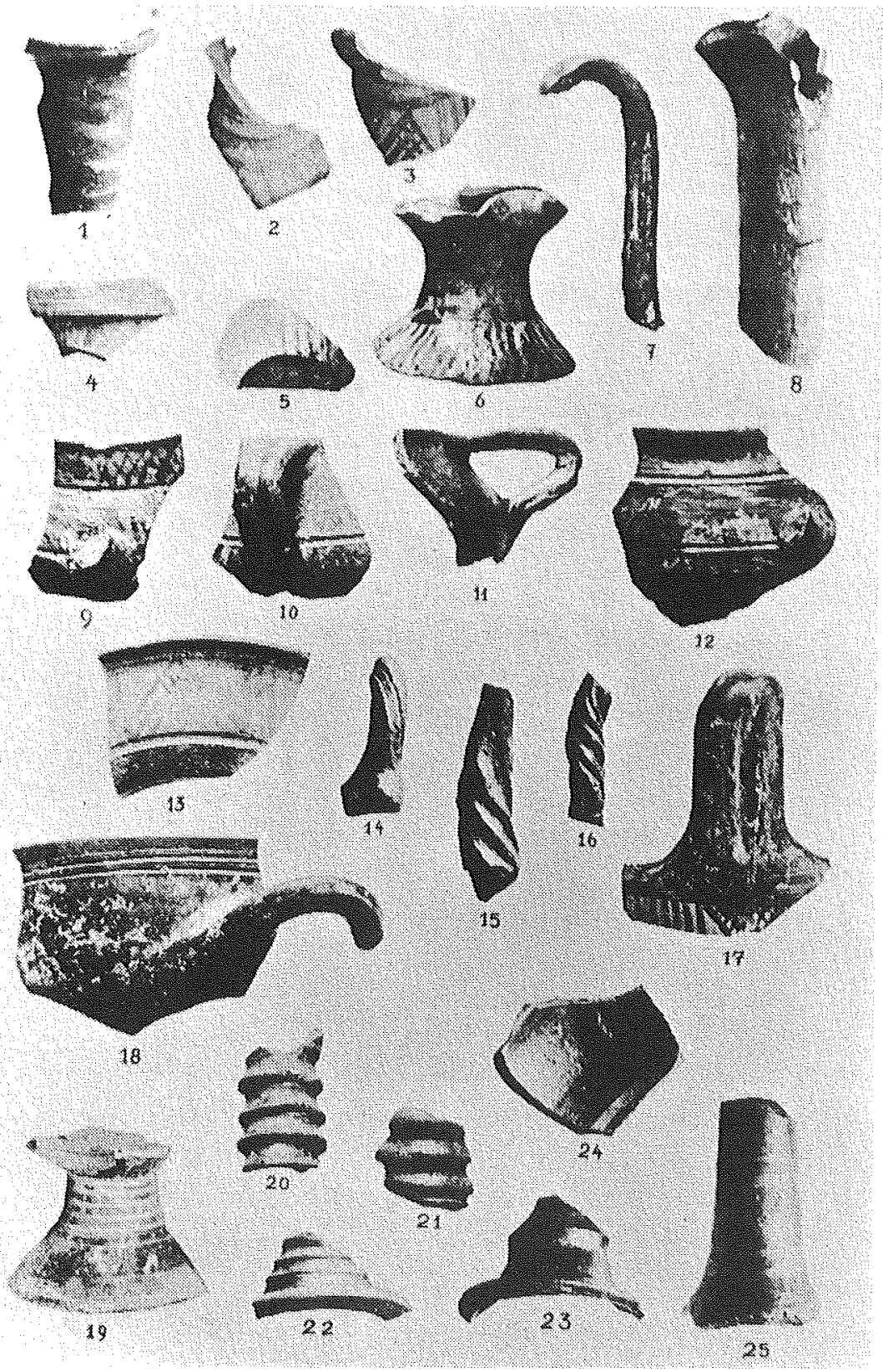

Pl. II : Trouvailles protogéométriques

d'après, E. BuschOR-W. VON MASSOW, in $\operatorname{MDAI(A),~} 52$ (1927), Pl. II 

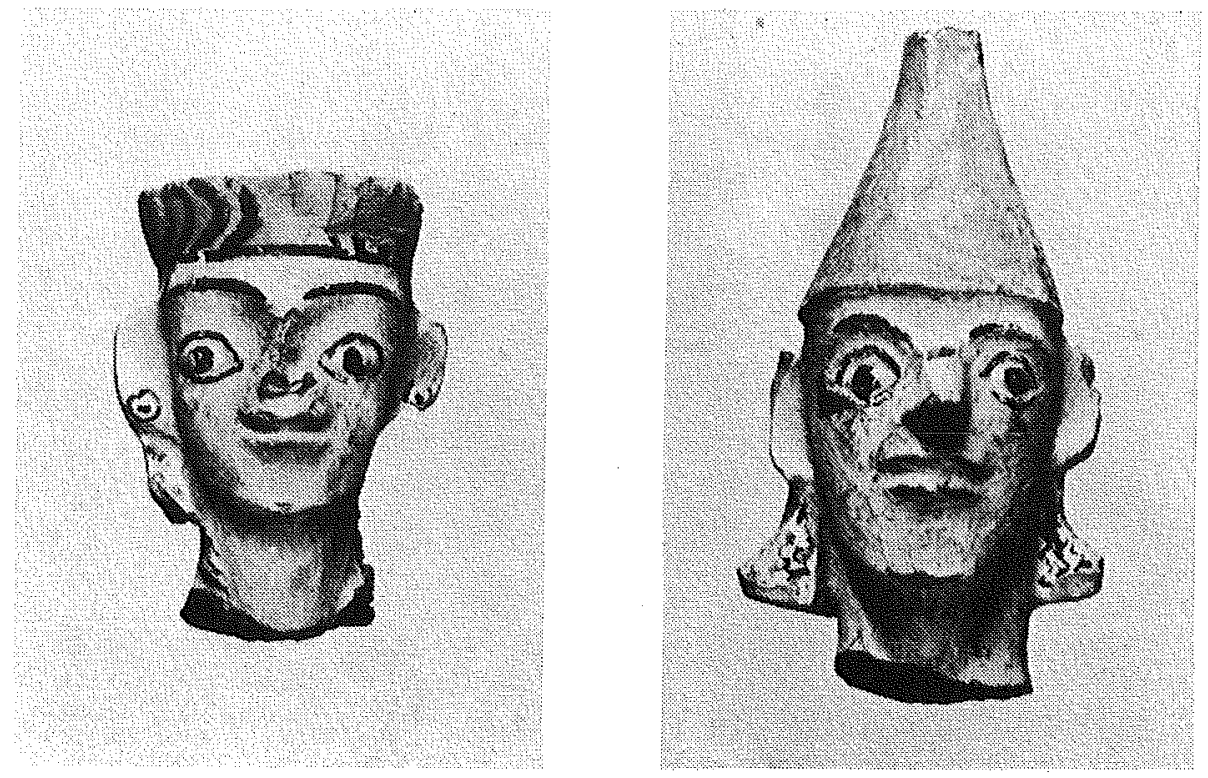

Pl. III : Têtes d'argile

d'après E. KuNZE, in $M D A I(A), 55$ (1930), Beilage XLII

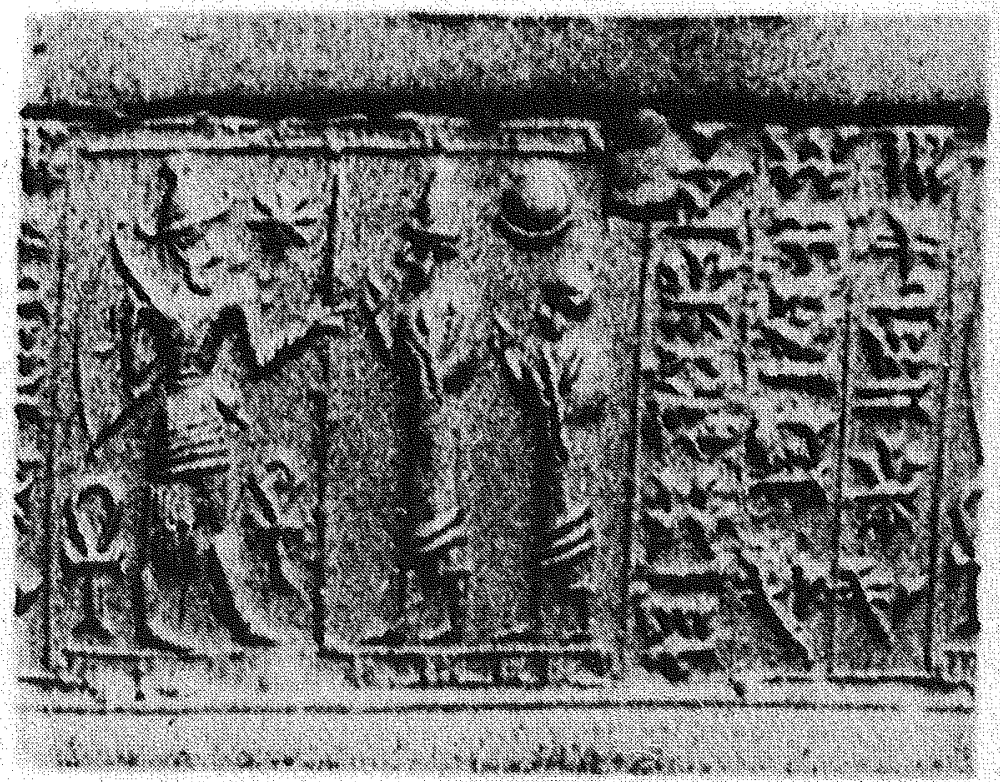

Pl. IV : Sceau syrien, d'après L. Delaporte, Catalogue des Cylindres Orien$\operatorname{taux}$, A919, Pl. 96: 17 


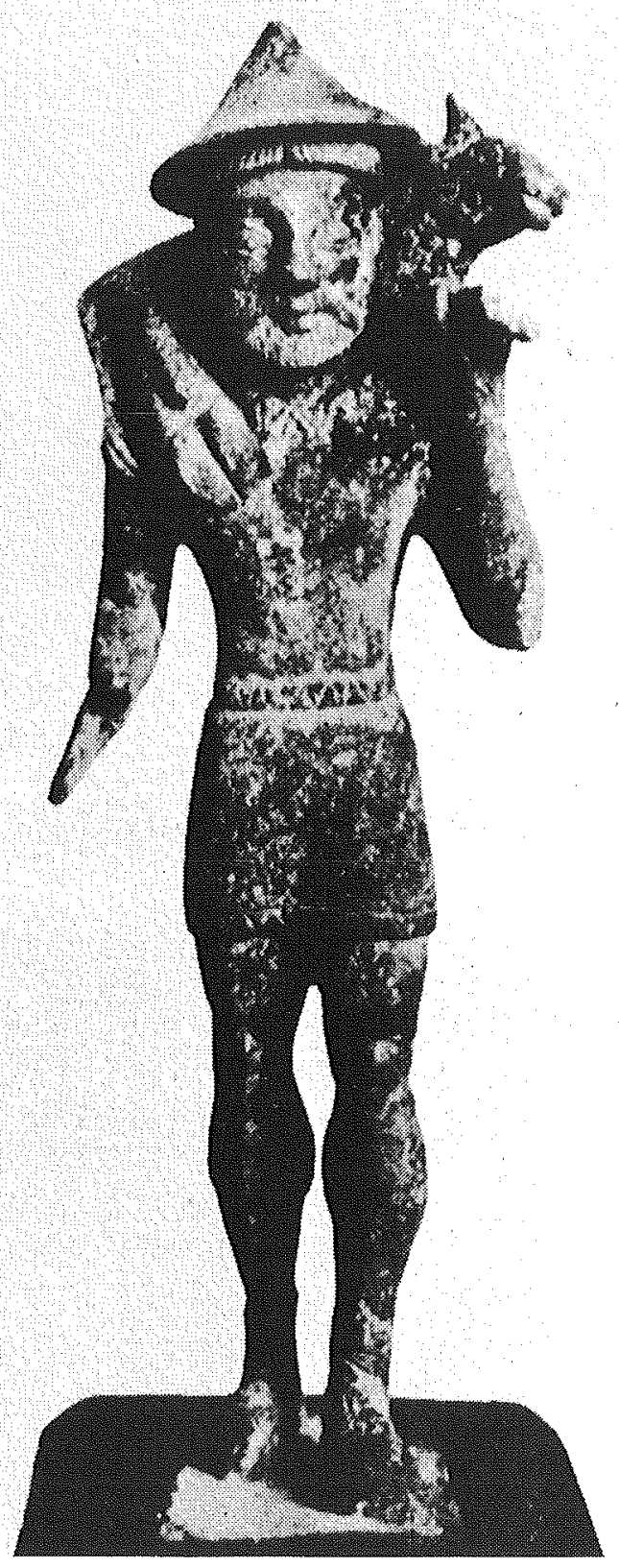

Pl. V : Statuette d'un berger arcadien d'après K. HADJIOANNOU, in Alasia I, fig. 2 